\title{
Thermal therapeutics
}

The development of temperature-inducible molecular bioswitches allows ondemand activation of synthetic genetic circuits in microbes.

Genetically engineered microbes could potentially be used to treat and diagnose disease. Such applications require activation of engineered synthetic microbial circuits with temporal and spatial precision. While chemical- and light-inducible methods for selective activation exist, they may be impractical for use in patients. This led Mikhail Shapiro and colleagues at the California Institute of Technology to develop temperature-inducible bioswitches that can precisely control synthetic circuits in animal tissues.

Shapiro and colleagues screened a panel of temperature-sensitive transcriptional repressors and heat-inducible promoters to identify switches that could effectively induce a reporter in response to increased temperature. Most promising were a transcriptional repressor from Salmonella typhimurium called TlpA and a temperature-sensitive variant of the bacteriophage $\lambda$ repressor referred to as TcI. Both could induce thirty-fold induction of a reporter gene and required only a small increase in temperature.

As different applications require activation at different temperatures, the authors fine tuned the activation threshold of each switch. They produced mutant variants of TlpA that were activated at 36 or $39^{\circ} \mathrm{C}$ instead of the original threshold of $43.5^{\circ} \mathrm{C}$. Likewise, they produced variants of $\mathrm{Tcl}$ activated at 38 or $42{ }^{\circ} \mathrm{C}$ rather than $39.5^{\circ} \mathrm{C}$. The switches could be coupled in a thermal logic circuit whereby different fluorescent reporters were expressed at different temperature ranges.

To test the viability of the switches for biotherapeutic applications, the authors used focal ultrasound, a technology that can specifically induce local changes in temperature even deep within tissues. The temperature increase activated reporter gene expression in Escherichia coli cells that were subcutaneously injected into mice. The authors envisage that such technology could be used to deliver treatments specifically at the site of a diseased organ. The switches were also used to activate reporter expression in response to fever-induced increases in host temperature, a feature that could be useful for diagnostic purposes. Finally, the authors also coupled TlpA to a gene encoding a bacterial toxin, thereby creating a kill switch. Such a switch could allow survival of engineered microbes at temperatures found in the gastrointestinal tract but would cause microbial death upon excretion, providing a strategy for biocontainment of engineered microbes.

\section{Richard Pattison}

\section{RESEARCH PAPERS}

Piraner, D. et al. Tunable thermal bioswitches for in vivo control of microbial therapeutics. Nat. Chem. Biol. http://dx.doi.org/10.1038/nchembio.2233 (2016). 